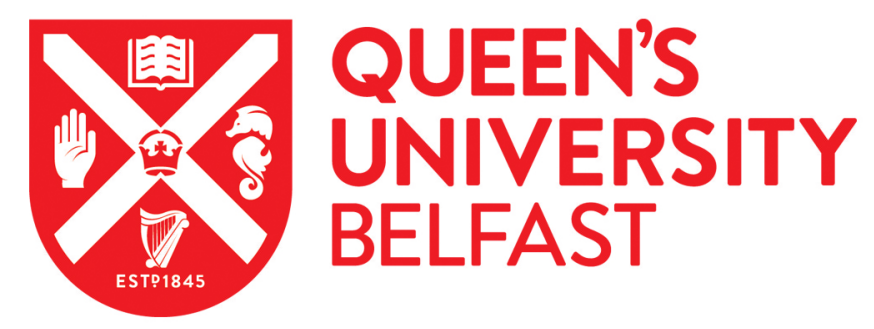

\title{
An eclipsing M-dwarf close to the hydrogen burning limit from NGTS
}

Acton, J. S., Goad, M. R., Casewell, S. L., Vines, J. I., Burleigh, M. R., Eigmüller, P., Nielsen, L. D., Gänsicke, B. T., Bayliss, D., Bouchy, F., Bryant, E. M., Gill, S., Gillen, E., Günther, M. N., Jenkins, J. S., McCormac, J., Moyano, M., Raynard, L. R., Tilbrook, R. H., ... Wheatley, P. J. (2020). An eclipsing M-dwarf close to the hydrogen burning limit from NGTS. Monthly Notices of the Royal Astronomical Society, 498(3), 3115-3124. https://doi.org/10.1093/mnras/staa2513

Published in:

Monthly Notices of the Royal Astronomical Society

Document Version:

Peer reviewed version

Queen's University Belfast - Research Portal:

Link to publication record in Queen's University Belfast Research Portal

Publisher rights

(C) 2020 Royal Astronomical Society.

This work is made available online in accordance with the publisher's policies. Please refer to any applicable terms of use of the publisher.

\section{General rights}

Copyright for the publications made accessible via the Queen's University Belfast Research Portal is retained by the author(s) and / or other copyright owners and it is a condition of accessing these publications that users recognise and abide by the legal requirements associated with these rights.

Take down policy

The Research Portal is Queen's institutional repository that provides access to Queen's research output. Every effort has been made to ensure that content in the Research Portal does not infringe any person's rights, or applicable UK laws. If you discover content in the Research Portal that you believe breaches copyright or violates any law, please contact openaccess@qub.ac.uk. 


\title{
An eclipsing M-dwarf close to the hydrogen burning limit from NGTS
}

\author{
Jack S. Acton, ${ }^{1 \star}$ Michael R. Goad, ${ }^{1}$ Sarah L. Casewell, ${ }^{1}$ Jose I. Vines, ${ }^{2}$ Matthew \\ R. Burleigh, ${ }^{1}$ Philipp Eigmüller, ${ }^{3}$ Louise D. Nielsen, ${ }^{4}$ Boris T. Gänsicke, ${ }^{5}$ Daniel \\ Bayliss ${ }^{5}$ François Bouchy, ${ }^{4}$ Edward M. Bryant, ${ }^{5}$ Samuel Gill, ${ }^{5}$ Edward Gillen, ${ }^{6,7} \dagger$ \\ Maximilian N. Günther, ${ }^{8}+$ James S. Jenkins, ${ }^{2,9}$ James McCormac, ${ }^{5}$ Maximiliano \\ Moyano, ${ }^{10}$ Liam R. Raynard, ${ }^{1}$ Rosanna H. Tilbrook, ${ }^{1}$ Stéphane Udry, ${ }^{4}$ Christopher \\ A. Watson, ${ }^{11}$ Richard G. West, ${ }^{5}$ Peter J. Wheatley, ${ }^{5}$ \\ ${ }^{1}$ School of Physics and Astronomy, University of Leicester, University Road, Leicester, LE1 7RH, UK \\ ${ }^{2}$ Departamento de Astronomía, Universidad de Chile, Camino el Observatorio 1515, Las Condes, Santiago, Chile \\ ${ }^{3}$ Institute of Planetary Research, German Aerospace Center, Rutherfordstrasse 2, 12489, Berlin, Germany \\ ${ }^{4}$ Geneva Observatory, University of Geneva, Chemin des Mailettes 51, 1290 Versoix, Switzerland \\ ${ }^{5}$ Department of Physics, University of Warwick, Gibbet Hill Road, Coventry CV4 7AL, UK \\ ${ }^{6}$ Astronomy Unit, Queen Mary University of London, Mile End Road, London E1 4NS, UK \\ ${ }^{7}$ Astrophysics Group, Cavendish Laboratory, J.J. Thomson Avenue, Cambridge CB3 OHE, UK \\ ${ }^{8}$ Department of Physics, and Kavli Institute for Astrophysics and Space Research, Massachusetts Institute of Technology, Cambridge, MA 02139, \\ ${ }^{9}$ Centro de Astrofísica y Tecnologías Afines (CATA), Casilla 36-D, Santiago, Chile \\ ${ }^{10}$ Instituto de Astronomía, Universidad Católica del Norte, Angamos 0610, 1270709, Antofagasta, Chile \\ 11 Astrophysics Research Centre, School of Mathematics and Physics, Queenẩ̆́́s University Belfast, Belfast, BT7 $1 N N$, UK
}

Accepted XXX. Received YYY; in original form ZZZ

\begin{abstract}
We present the discovery of NGTS J0930-18, an extreme mass ratio eclipsing Mdwarf binary system with an early M-dwarf primary and a late M-dwarf secondary close to the hydrogen burning limit. Global modelling of photometry and radial velocities reveals that the secondary component (NGTS J0930-18 B) has a mass of $\mathrm{M}_{*}=0.0818_{-0.0015}^{+0.0040} \mathrm{M}_{\odot}$ and radius of $\mathrm{R}_{*}=0.1059_{-0.0021}^{+0.0023} \mathrm{R}_{\odot}$, making it one of the lowest mass stars with direct mass and radius measurements. With a mass ratio of $\mathrm{q}$ $=0.1407_{-0.017}^{+0.0065}$, NGTS J0930-18 has the lowest mass ratio of any known eclipsing M-dwarf binary system, posing interesting questions for binary star formation and evolution models. The mass and radius of NGTS J0930-18 B is broadly consistent with stellar evolutionary models. NGTS J0930-18 B lies in the sparsely populated mass radius parameter space close to the substellar boundary. Precise measurements of masses and radii from single lined eclipsing binary systems of this type are vital for constraining the uncertainty in the mass-radius relationship - of importance due to the growing number of terrestrial planets being discovered around low mass stars.
\end{abstract}

Key words: binaries: eclipsing, stars: low-mass

\section{INTRODUCTION}

Eclipsing binary stars are of vital importance in the field of stellar structure. These are the only objects from which we are able to get accurate mass-radius measurements of stars to test against model predictions. This is particularly

\footnotetext{
$\star$ E-mail:ja466@le.ac.uk

$\dagger$ Winton Fellow

$\ddagger$ Juan Carlos Torres Fellow
}

relevant for low mass stars (known as M-dwarfs). Stars with masses below $0.25 M_{\odot}$ are the most common stellar objects (Henry et al. 2006) but despite this the physics governing them remains relatively poorly understood. In particular, the mass-radius relation for low mass stars is poorly constrained when compared with theoretical models (Parsons et al. 2018).

Previous studies have shown that model predictions for masses and radii of $\mathrm{M}$-dwarfs can differ from measured values by up to 10 per cent (Feiden \& Chaboyer 2012; Ter-

(C) 2020 The Authors 
rien et al. 2012). This discrepancy is most likely due to magnetic activity induced by interactions in short-period tidally-locked binary systems (Ribas 2006; Chabrier et al. 2007). However, this is not entirely clear due to the existence of longer period systems, which we would not expect to be tidally locked, that show the same over sizing as these short period systems (Doyle et al. 2011, Irwin et al. 2011), as well as short period systems that show good agreement with models (Blake et al. 2008). This is further complicated by the fact that it is expected that fully convective stars (those with masses $<0.35 M_{\odot}$ ) should show less inflation due to the nature of their atmospheres (Kraus et al. 2011).

It is then vital that we are able to further constrain models for low mass stars by obtaining direct measurements of stellar masses and radii. This has motivated the search for examples of low-mass eclipsing binaries (also known as EBLMs) that have provided accurate mass and radius measurements for a large number of M-dwarf stars across a range of masses (e.g Triaud et al. 2012; GÃşmez Maqueo Chew et al. 2014; von Boetticher et al. 2017; Triaud et al. 2017; von Boetticher et al. 2019; Gill et al. 2019b). However the lowest masses, between $0.1 M_{\odot}$ and hydrogen burning limit at $\sim 0.07 M_{\odot}$, remains relatively sparsely sampled.

When characterising exoplanet systems, accurate knowledge of the host star's parameters is crucial as these are used to determine the corresponding values for the planet. Uncertainties in the stellar values could lead to over or underestimation of discovered planetary masses and radii. This is of further importance as some of the most interesting planetary systems have been discovered around low mass stars (e.g., Gillon et al. 2017; Günther et al. 2019; Kostov et al. 2019). The smaller radii of these stars means that small planets produce transits of a much greater depth than the same planet occulting a larger star, making them much easier to detect in transit surveys. For this reason many modern transiting exoplanet surveys are designed to target such low mass stars (e.g., TRAPPIST (Gillon et al. 2011), SPECULOOS (Delrez et al. 2018)).

Additionally, observations of rare types of eclipsing binary systems can provide insights into star formation. For example, Wisniewski et al. 2012 proposed that there should be a lack of binaries with orbital periods less than 100 days with highly unequal mass components. There are examples of so called Extreme Mass Ratio Binaries (EMRBs) comprising a large star (typically A or B spectral type) with an M-dwarf companion (e.g., Stevens et al. 2019), however the vast majority of eclipsing M-dwarf binaries are systems of roughly equal mass (Delfosse et al. 2004). Laithwaite \& Warren 2020 surveyed a large sample of late M-dwarf binaries and found that they are almost exclusively equal mass systems. This is possibly a formation effect. Bouchy et al. 2011 propose that stars with spectral types later than $\mathrm{G}$ have disk braking strong enough to cause low mass short period companions to migrate inwards and become engulfed. Therefore we would expect extreme mass ratio binaries of two low mass stars to be rare.

In this paper we present the discovery of NGTS J093016-185033.6 (hereafter NGTS J0930-18), a highly unequal mass ratio ( $q=0.14075)$ eclipsing $M$-dwarf binary in which the secondary (NGTS J0930-18 B) is a very low mass star just above the classical hydrogen burning limit of $70 \mathrm{M}_{\mathrm{J}}$ (Dieterich et al. 2014). We make use of follow-up pho- tometric and spectroscopic observations to determine accurate masses and radii for the star, which lies in a region of parameter space with few direct measurements. This discovery will aid in further constraining the lowest end of the stellar mass-radius relationship.

\section{OBSERVATIONS}

NGTS J0930-18 was initially discovered using photometry from NGTS (Wheatley et al. 2018). Follow-up observations were performed with the Sutherland High Speed Optical Cameras (SHOC) (Coppejans et al. 2013) on the South African Astronomical Observatory (SAAO) 1-m telescope. This photometry was then used in conjunction with observations from the Transiting Exoplanet Survey Satellite (TESS, Ricker et al. 2014). We obtained high resolution spectra with HARPS (mounted on the ESO 3.6m, Mayor et al. 2003) to determine the mass of the companion. These observations are detailed in Table 1 and described below.

\subsection{NGTS}

NGTS J0930-18 was initially identified in photometry from the Next Generation Transit survey (hereafter NGTS; Wheatley et al. 2018). NGTS is a wide-field photometric survey consisting of an array of 12 fully automated $20 \mathrm{~cm}$ telescopes operating at ESO's Paranal observatory in Chile. The facility has been operational since early 2016, and is optimised for observations of K- and M-type stars, with sensitivity in the 520 to $890 \mathrm{~nm}$ wavelength range. NGTS has a wide field of view (instantaneously covering $96 \mathrm{sq}$ deg) and delivers high cadence (every $\sim 13$ seconds) photometry with high precision (1mmag per hour for an $\mathrm{I}=14$ magnitude star).

The optimization of NGTS for precise photometry of late spectral-type stars has allowed it to make several discoveries of interesting M-dwarf systems. These include the discovery the most massive planet orbiting an M-dwarf (Bayliss et al. 2018) as well as the shortest period brown dwarf around a main sequence star (in this case an early M-dwarf) (Jackman et al. 2019). NGTS has also discovered M-dwarfs in double-lined eclipsing binaries (Casewell et al. 2018; Acton et al. 2020) and low mass stars in long period orbits around stars of other spectral types (Gill et al. 2019a; Lendl et al. 2019). M-dwarf stars continue to be a key focus of the NGTS science program.

NGTS J0930-18 was observed during the 2016 NGTS observing season. The field containing the system was observed for 156 nights between 2016 October $17^{\text {th }}$ and 2017 June $21^{\text {st }}$, and in total we obtained $185,22710 \mathrm{~s}$ exposure science images. The magnitude of the system in various bandpasses, as well as positional information, is provided in Table 2. To allow for the detection of the system, the light curve was first cleaned using an implementation of the SysRem algorithm (Tamuz et al. 2005). Periodic signals which do not show a typical transit shape (such as those caused by stellar variability) were then automatically detected and removed. After cleaning the eclipses were detected using ORION (see Wheatley et al. 2018 for more information), an implementation of the BLS algorithm (Kovács et al. 2016).

ORION also calculated some initial parameters for the 
Table 1. Summary of observations.

\begin{tabular}{ccccccc}
\hline Observation type & Telescope & Band & Cadence & Total integration time & Period & Notes \\
\hline Photometry & NGTS & $520-890 \mathrm{~nm}$ & $13 \mathrm{~s}$ & 156 nights & $21 / 04 / 16-22 / 12 / 16$ & 14 full eclipses \\
Photometry & SAAO & I & $30 \mathrm{~s}$ & 3 hours & $20 / 12 / 18$ & Single Observation \\
Photometry & SAAO & g' & $10 \mathrm{~s}$ & 2.16 hours & $29 / 01 / 19$ & Single Observation \\
Photometry & TESS & $600-1000 \mathrm{~nm}$ & $1800 \mathrm{~s}$ & 28 days & $02 / 02 / 19-27 / 02 / 19$ & 10 eclipses in total \\
Spectroscopy & HARPS & $378-691 \mathrm{~nm}$ & 45 minutes & 4.5 hours & $11 / 04 / 19-08 / 06 / 19$ & Six RV Points, EGGS mode \\
\hline
\end{tabular}

Table 2. Stellar Properties and colour magnitudes for NGTS J0930-18 obtained from Gaia DR2 (Gaia Collaboration et al. 2018), NGTS (Wheatley et al. 2018) TIC v8 (Stassun et al. 2019) and 2MASS (Skrutskie et al. 2006).

\begin{tabular}{ccc} 
Property & Value & Source \\
\hline Gaia I.D. & DR2 5678383069566263552 & Gaia \\
TIC I.D. & 176772671 & TIC v8 \\
R.A. (J2000) & $09: 30: 16.0$ & NGTS \\
Dec (J2000) & $-18: 50: 33.62$ & NGTS \\
$\mu_{\alpha}\left(\right.$ mas yr $\left.^{-1}\right)$ & $-30.528 \pm 0.255$ & Gaia \\
$\mu_{\delta}\left(\right.$ mas yr $\left.^{-1}\right)$ & $18.0662 \pm 0.234$ & Gaia \\
Parallax (mas) & $4.392 \pm 0.140$ & Gaia \\
$G$ & 14.8357 & Gaia \\
NGTS & 13.98 & NGTS \\
TESS & 13.8995 & 2MASS \\
$V$ & 15.529 & 2MASS \\
$J$ & 12.701 & 2MASS \\
$H$ & 12.06 & 2MASS \\
$K_{S}$ & 11.869 & 2MASS \\
\hline
\end{tabular}

system, identifying a $2.3 \%$ depth eclipse with a period of 1.33 days, which allowed the object to be identified as a candidate exoplanet. When phase-folded on the ORION identified period, we saw no evidence for a secondary eclipse at phase 0.5 , implying that the companion must have a surface brightness which is significantly less than the primary star. Due to the lack of secondary eclipse and a transit depth which was consistent with a planetary companion, the object was followed-up photometrically and spectroscopically, where it was determined that the eclipsing object was in fact a low mass stellar companion.

Four Gaia DR2 (Gaia Collaboration et al. 2018) sources are present in the $15^{\prime \prime}$ radius photometric aperture used by the NGTS pipeline, consequently we could not be certain which star was the source of the eclipses identified by ORION (see Figure 1). Two of these stars are fainter than $G=18$ (Gaia band), and therefore contribute negligible flux and cannot be the source of the signal seen in the NGTS data. The remaining two objects have the same parallax and proper motion, and therefore are a physically related pair, with the eclipse event occurring on one of these. To identify the source of the eclipse we used the centroid vetting technique described in Günther et al. (2017) which allows the measurement of extremely small shifts in the flux centroid during eclipse. Using this technique we identified a significant centroid shift indicating the southern star to be the eclipse source (Gaia ID - 5678383069566263552) - see Figure 1.

NGTS observations captured 14 full eclipses of the system in total, as well as a large number of partial eclipses at

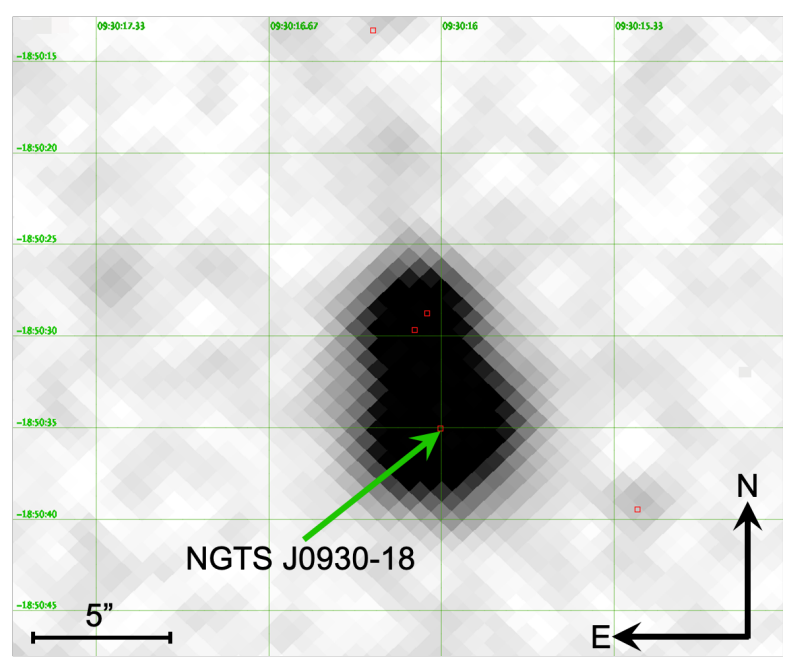

Figure 1. Digital Sky Survey (DSS) optical image of NGTS J0930-18. The red squares indicate the positions of identified Gaia DR2 sources. Four sources are present in the NGTS aperture which could contribute to the detection. NGTS J0930-18 is the larger southern star, which is physically associated with the smaller northern star.

the start or end of observing nights. The NGTS discovery lightcurve is shown in Figure 2.

\subsection{TESS}

NGTS J0930-18 was observed in Sector 8 of the TESS mission (TIC-176772671, T=13.98), which occurred between 2019 February $2^{\text {nd }}$ and 2019 February $27^{\text {th }}$. These observations consist of standard full frame images taken with a cadence of 30 minutes. We extracted the photometry for NGTS J0930-18 from the full frame images of CCD 3 of camera 2. We used a custom aperture selected based on a flux threshold to minimise blending. However, due to the large TESS pixel size the blending is still greater than in the NGTS lightcurve. A floating median was applied to mask out systematic flux drops due to spacecraft effects. Further details of this method can be found in Gill et al. 2020 .

The signal is clearly detected in the TESS data, with a total of seven eclipses captured by the TESS observations. A BLS search of the TESS data alone identifies a similar periodicity to the NGTS data, further validating this detection. The TESS lightcurve is shown in Figure 3. As for the NGTS lightcurve, we see no evidence of a secondary eclipse, or significant out of transit variation in the lightcurve. 


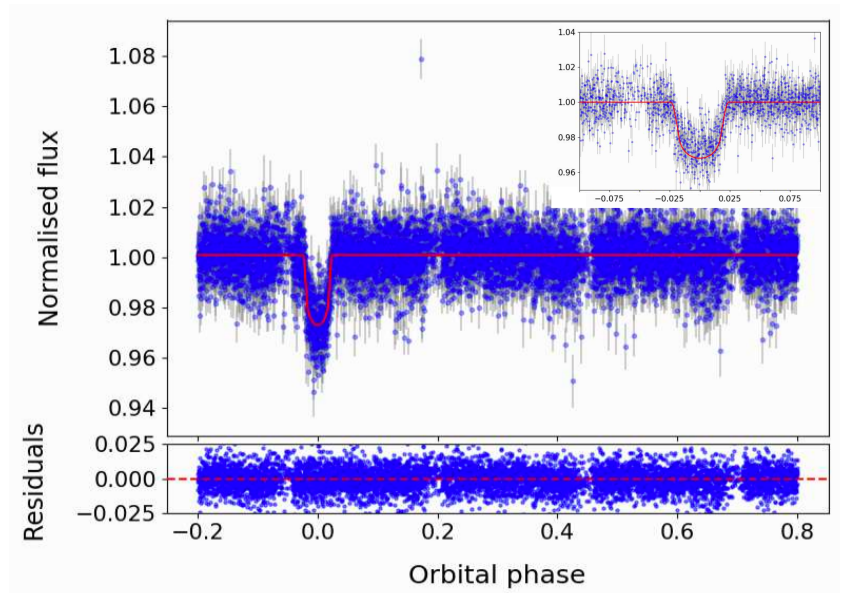

Figure 2. NGTS photometry of NGTS J0930-18 folded on a period of 1.33265 days and binned to 5 minutes. The red line shows the model fit obtained from joint modelling of photometric and spectroscopic data.Note that there is no obvious secondary eclipse at phase 0.5 . Inset - zoomed in plot of the primary eclipse of the system.

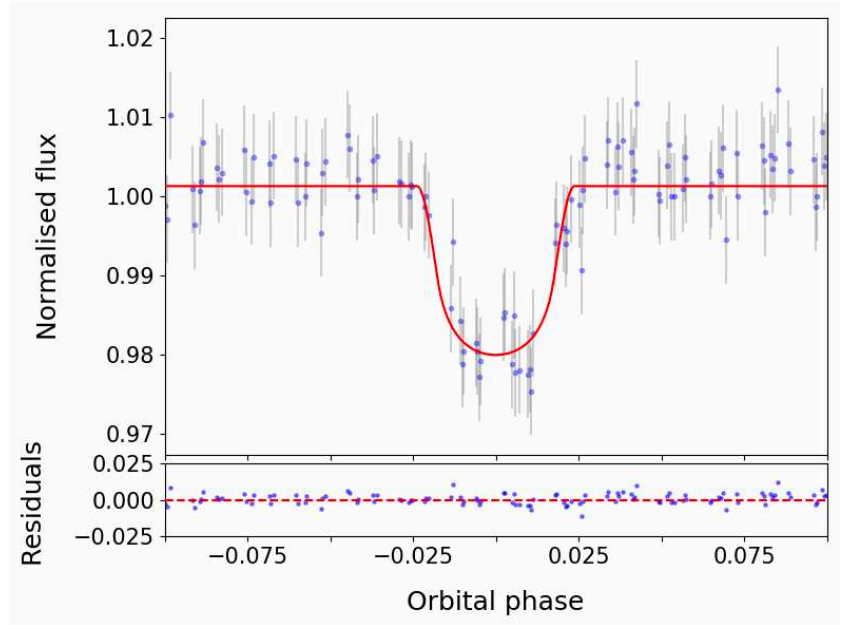

Figure 3. TESS 30-minute cadence photometry of the eclipse of NGTS J0930-18 folded on the period of 1.33265 days. The red line shows the model fit obtained from joint modelling of photometric and spectroscopic data.

\subsection{SAAO Photometry}

We obtained photometry of NGTS J0930-18 using the SAAO 1-m telescope equipped with the SHOC instrument (Coppejans et al. 2013) on 2018 December $20^{\text {th }}$ and 2019 January $29^{\text {th }}$ in I and g' bands. The aim of the observation was to confirm transit depth and width and to refine the ephemeris for the system. Additionally, by obtaining multi-colour photometry we can check for any wavelength dependent eclipse depth differences. The observations would also allow us to confirm which star is the source of the transit signal as they should be spatially resolved given the plate-scale of SHOC on the SAAO 1-m telescope $\left(0.167^{\prime \prime}\right.$ per pixel, binned by a factor 4). The I band observations consisted of $360 \times 30 \mathrm{sec}$

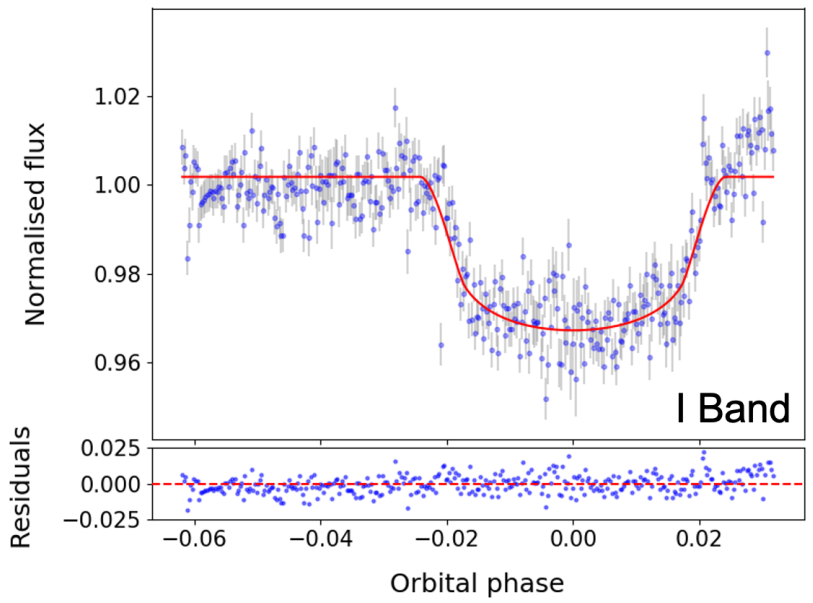

Figure 4. SAAO $1 \mathrm{~m} / \mathrm{SHOC} I$-band photometry from the night 2018 December $20^{\text {th }}$ showing a primary eclipse of NGTS J0930-18 plotted in phase. The red line shows the model fit obtained from joint modelling of photometric and spectroscopic data.

exposures for a total observation time of 3 hours. The g' band observations were $770 \times 10 \mathrm{sec}$ exposures for a total observing time of 7700 seconds ( $\equiv 2.13$ hours).

Standard bias and flat fielding corrections were applied to the data using the local SAAO SHOC pipeline, which is driven by PYTHON scripts running IRAF tasks (PYFITS and PYRAF). Aperture photometry was performed using the STARLINK package AUTOPнотом, which also measured and subtracted the sky background. The number of comparison stars and size of the aperture were chosen to minimise the RMS scatter outside of the eclipse. For both sets of observations we used a 4 pixel $\left(2.67^{\prime \prime}\right)$ aperture with 2 stars used for comparison.

Both of these observations clearly detect the eclipse. The I band observations detect a full eclipse of the system, whereas the g' band observations capture the flux during eclipse as well as the egress. Additionally, the two brighter Gaia sources were able to be resolved. From this we were able to confirm that the eclipse occurred on the southern star, the brighter of the two possible eclipse sources, consistent with the result from centroid analysis. The lightcurves for each filter are shown in Figure 4 and Figure 5. We note that there is not a significant difference in the eclipse depth between the two bands.

\subsection{Radial Velocity Measurements}

To determine the mass of the eclipsing object, NGTS J093018 was observed with the HARPS spectrograph on the ESO $3.6 \mathrm{~m}$ telescope (Mayor et al. 2003) under programme 0103.C-0719 (PI. Bouchy). Due to the optical faintness of the object $(\mathrm{V}=15.30)$ we used the high efficiency (EGGS) mode, which uses a larger fibre in order to improve $\mathrm{S} / \mathrm{N}$ at the expense of a modest reduction in spectral resolution. A total of six observations were taken between 2019 April $11^{\text {th }}$ and 2019 June $8^{\text {th }}$, with all exposure times being 2700 seconds.

Observations were reduced using the standard HARPS 


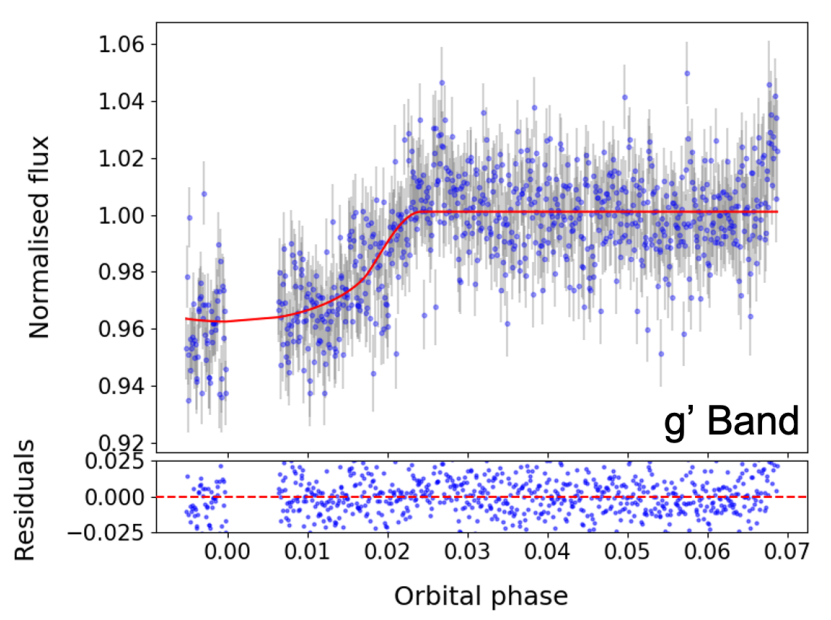

Figure 5. SAAO $1 \mathrm{~m} / \mathrm{SHOC} g^{\prime}$ filter photometry from the night 2019 January $29^{\text {th }}$ of a primary eclipse of NGTS J0930-18 plotted in phase. The red line shows the model fit obtained from joint modelling of photometric and spectroscopic data. The data gap starting near phase 0.0 is due to passing clouds during the observations.

data reduction pipeline. The spectra were cross-correlated with a template G2 stellar mask and the cross correlation function (CCF) derived to determine the radial velocity of the star for each observation epoch. We used a G-type mask rather than an M-type as the star is rapidly rotating, and M-type masks struggle to deal with this. A single peak with large ( $\mathrm{K} \sim 22 \mathrm{~km} / \mathrm{s}$ scale) velocity shift was detected, consistent with a low mass stellar companion. The radial velocities show a clear periodicity in phase with the period determined from photometric observations. The phase folded radial velocity curve is shown in Figure 6. We checked for correlation between bisector span and radial velocity and found no evidence for such correlation. The full radial velocity measurements are given in Table 3 .

\section{RESULTS}

\subsection{Primary Star Parameters}

\subsubsection{Spectral Typing}

In order to determine the spectral type of the primary star, NGTS J0930-18 A, we performed a template matching procedure. The HARPS spectra were wavelength shifted and then co-added to create a single higher signal-to-noise spectrum for analysis (SNR of 17). The spectral type was determined by comparing this spectrum with templates derived from Sloan Digital Sky Survey (SDSS) spectra covering a wide range of spectral types using the PYHAMMER code (Kesseli et al. 2017) a python implementation of the HAMMER spectral classification routine (Covey et al. 2007). PYHAMMER computes a chi-squared value that compares 34 spectral indices for each template, which are weighted relative to uncertainties in the individual observed spectra, to the corresponding values for the input spectrum. The spectral type which produces the minimum chi-squared value is taken as the assumed spectral type of the input star. The

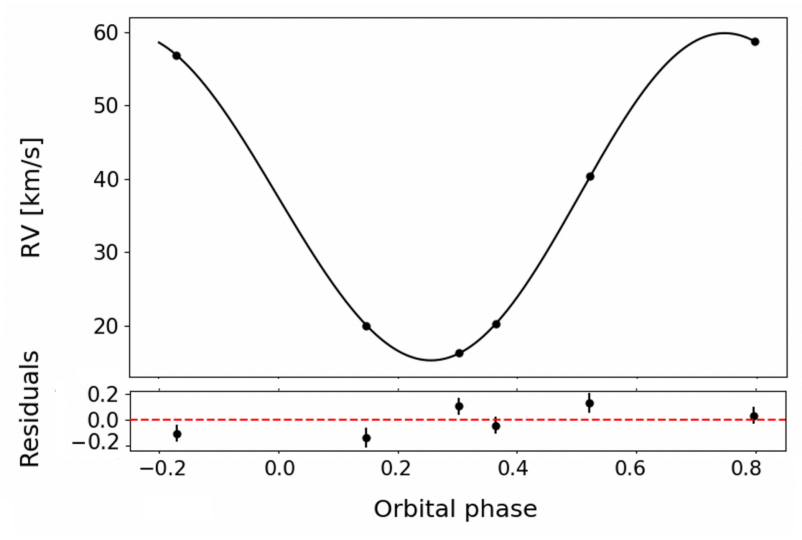

Figure 6. Phase folded radial velocity curve for NGTS J093018 with black circles showing the six radial velocity measurements taken by HARPS between 2019 April $11^{\text {th }}$ and 2019 June $8^{\text {th }}$. Radial velocities were phase folded on a period of 1.33265 days. The solid black line shows the model fit to the radial velocities obtained from global modelling of the system. Fit residuals (data-model) are shown in the lower panel.

best matching template to our combined HARPS spectrum is that of an M0V star with $[\mathrm{Fe} / \mathrm{H}] \sim 0$. We note however that the metallicity is not strongly constrained by these fits, with higher and lower metallicity templates also showing reasonable agreement with our spectrum.

\subsubsection{Spectral Analysis}

An initial estimate of the stellar parameters for NGTS J0930-18 A was obtained using SPECMATCH-EMP (Yee et al. 2017). These parameters would be used as priors when fitting the spectral energy distribution of the star to improve the quality of the result. SPECMATCH-EMP characterises stars based on their optical spectra, making use of a substantial library of high resolution $(\mathrm{R} \sim 55,000)$, high $\mathrm{S} / \mathrm{N}(>100)$ spectra obtained using Keck/HIRES. These high quality template spectra are used to classify an input spectrum (in this case the combined HARPS spectrum of NGTS J0930-18 A).

SPECMATCH-EMP effectively performs a two step process. First, the input spectrum is shifted so that it is on the same wavelength scale as the library (template) spectra. This is achieved by performing a cross correlation between the input spectrum and several reference spectra in turn for a predetermined wavelength region. The reference spectrum which gives the largest cross correlation peak is then used to shift the entire spectrum.

Once the HARPS spectrum has been shifted to the appropriate wavelength range, the matching procedure is applied. The input spectrum is compared with every other star in the library (for a given wavelength range, e.g., $\mathrm{Mg} \mathrm{b}$ triplet). $V \sin i$ is allowed to float, and a spline is fit to the continuum. The best matching stars to the input spectrum are identified using a chi-squared analysis. Linear combinations of the best matching spectra are then used to obtain an even better match than the individual best matching spectra alone. A weighted average of the library parameters is then taken and used to determine the properties of the target star. For NGTS J0930-18 A these are given in Table 4. 
Table 3. Radial Velocities for NGTS J0930-18

\begin{tabular}{ccccc}
\hline $\begin{array}{c}\text { BJD TDB } \\
(-2,450,000)\end{array}$ & $\begin{array}{c}\text { RV } \\
(\mathrm{km} / \mathrm{s})\end{array}$ & $\begin{array}{c}\text { RV error } \\
(\mathrm{km} / \mathrm{s})\end{array}$ & $\begin{array}{c}\text { FWHM } \\
(\mathrm{km} / \mathrm{s})\end{array}$ & $\begin{array}{c}\text { Contrast } \\
(\%)\end{array}$ \\
\hline 8584.6529 & 20.269 & 0.064 & 33.650 & 12.602 \\
8585.6940 & 20.025 & 0.077 & 33.957 & 12.045 \\
8586.6039 & 56.840 & 0.065 & 32.117 & 12.036 \\
8638.5344 & 58.792 & 0.062 & 32.464 & 11.472 \\
8640.5395 & 16.267 & 0.065 & 32.790 & 12.812 \\
8643.4978 & 40.395 & 0.074 & 33.058 & 12.180 \\
\hline
\end{tabular}

Table 4. NGTS J0930-18 A Stellar parameters derived from SPECMATCH-EMP and ARIADNE. For the parameters derived by ARIADNE, the first error is the statistical error whilst the second is a systematic error calculated from the maximum difference between the average value and the values produced by the individual theoretical models

\begin{tabular}{ccc}
\hline Parameter & SPECMATCH-EMP & ARIADNE \\
\hline Teff $(\mathrm{K})$ & $4069 \pm 70$ & $3982_{-16}^{+15} \pm 64$ \\
Logg $\left(\mathrm{cms}^{-2}\right)$ & $4.67 \pm 0.12$ & $4.687_{-0.055}^{+0.055} \pm 0.045$ \\
Radius $\left(\boldsymbol{R}_{\odot}\right)$ & $0.62 \pm 0.10$ & $0.584_{-0.0103}^{+0.0094} \pm 0.020$ \\
{$[\boldsymbol{F} \boldsymbol{e} / \boldsymbol{H}]$} & $-0.01 \pm 0.09$ & $-0.012_{-0.046}^{+0.041} \pm 0.085$ \\
Mass $\left(\boldsymbol{M}_{\odot}\right)$ & $0.62 \pm 0.08$ & $0.580_{-0.0063}^{+0.0092} \pm 0.017$ \\
Age $(\mathrm{Gyr})$ & $9.89 \pm 0.17$ & $9.20_{-5.31}^{+2.20} \pm 3.63$ \\
Distance $(\mathrm{pc})$ & - & $223.5_{-3.0}^{+3.5} \pm 4.5$ \\
\hline
\end{tabular}

Additionally we measured the projected stellar rotation velocity $(\mathrm{v} \sin (\mathrm{i}))$ by fitting synthesised spectra using iSpec (Blanco-Cuaresma et al. 2014). We fit only for $\mathrm{v} \sin (\mathrm{i})$, fixing the other values to those obtained from SPECMATCH-EMP. This provides a value for the projected stellar rotation velocity of $30.17 \mathrm{kms}^{-1}$, showing that the star is rapidly rotating. Given the inclination determined in 3.2 , this is consistent with the star being in a state of spin orbit synchronisation.

\subsubsection{SED Fitting}

We fit the spectral energy distribution (hereafter, SED) of NGTS J0930-18 A using ARIADNE (Vines \& Jenkins 2020). ARIADNE is a python tool which fits catalogue photometry to various atmospheric model grids. We fitted model grids from Phoenix v2 (Husser et al. 2013), BT-Settl, BT-Cond, BTNextGen (Allard et al. 2012; Hauschildt et al. 1999), Castelli \& Kurucz (2004), and Kurucz (1993). These were then convolved with various filter response functions.

Each model SED is created by interpolating the model grids in $\mathrm{T}_{\mathrm{eff}}-\log g-[\mathrm{Fe} / \mathrm{H}]$ space. We also used distance, radius and extinction in the $V$ band as model parameters. Additionally we include an excess noise term for each set of photometry to account for any underestimation of uncertainties. We applied priors for $\mathrm{T}_{\mathrm{eff}}, \log g$ and $[\mathrm{Fe} / \mathrm{H}]$ derived from the SPECMATCH analysis of the stacked HARPS spectrum (see section 3.1.2). Priors for radius and distance were taken from Gaia DR2 and $A_{V}$ was limited to the maximum line of sight value from the Schlegel, Finkbeiner \& Davis
(SFD) galactic dust map (Schlegel et al. 1998; Schlafly \& Finkbeiner 2011). Excess noise parameters were normally distributed around zero with a variance equal to five times the size of the reported uncertainty.

Parameter estimation was performed using dynesty's nested sampler for parameter estimation and calculating the bayesian evidence for each model (Speagle 2019). ARIADNE then calculates the weighted average of each parameter using the relative probabilities of each of the fitted models. A mass estimate is then computed using MIST isochrones (Choi et al. 2016). A detailed explanation of ARIADNE is given in Vines \& Jenkins (2020). The parameters for NGTS J0930-18 A derived by ARIADNE are given in Table 4 and a corner plot showing the posterior distribution of the key parameters is shown in Figure 7.

We note that the method employed by ARIADNE leads to results with a remarkable degree of precision. This is a result of the mathematical treatment of the posterior parameter distribution. The distribution of each model is averaged, using the relative probabilities of each model as weights. This is determined using the following equation

$P\left(\theta_{i}\right)=\sum_{n=1}^{N} P\left(\theta \mid X, M_{n}\right) P\left(M_{n} \mid X\right)$

Where $\theta_{i}$ is the parameter to be averaged, $P\left(\theta \mid X, M_{n}\right)$ is the posterior distribution derived using Bayes Theorem and $P\left(M_{n} \mid X\right)$ is the Bayesian evidence of the individual model. This probability is used as a weight when averaging over the full set of models.

This results in higher precision than would be obtained by the use of any one model alone. The increased precision obtained by averaging over posterior distributions can be seen in Figure 8, which shows the probability density function for the derived stellar radius. Preliminary testing has shown that ARIADNE obtains great accuracy compared to radii derived by interferometry. This is particularly important due to the direct dependence of the mass and radius of the companion on that of the host.

To account for the effect of underestimating uncertainties when averaging over models, we also calculate a systematic error for the parameters derived by ARIADNE. As in Southworth et al. (2015), we determine this by fitting the SED with each model individually, and taking the largest difference between these individual values and the average value as the systematic error.

The values obtained with ARIADNE are more precise, but still consistent with those obtained by SPECMATCH-EMP. These parameters are roughly consistent with an early M- 


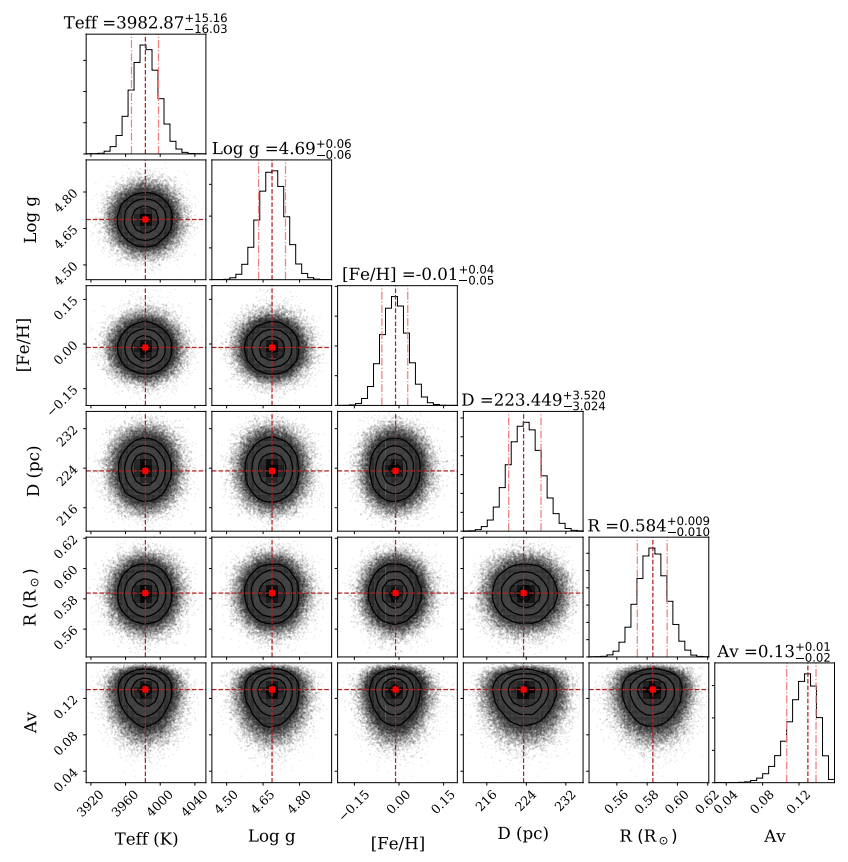

Figure 7. Cornerplot showing Key Primary Star parameters derived using ARIADNE in section 3.1.3

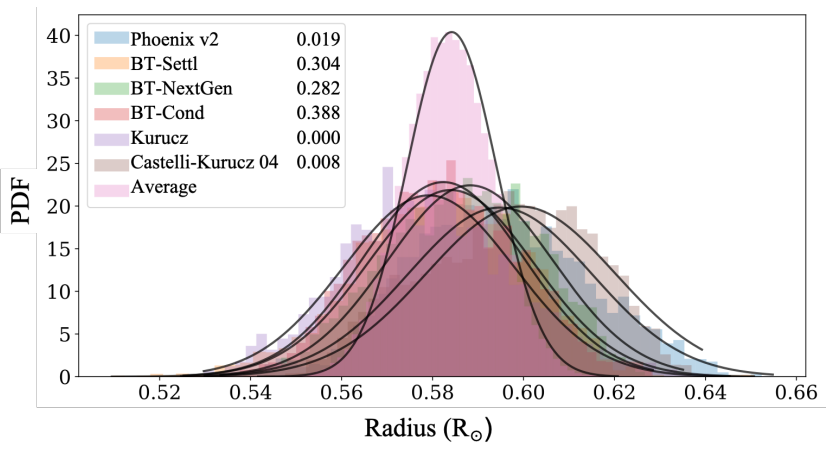

Figure 8. Histogram showing the probability distribution for the radius of NGTS J0930-18 A for each atmospheric model. The model probabilities are then used as weights to compute an overall average estimation, shown in pink, which has the best model value and uncertainty for the parameter.

dwarf star of spectral type M0V, as determined in $§ 3.1 .1$. We adopt the parameters derived by ARIADNE to derive the orbital solution as detailed in $§ 3.2$.

\subsection{Global Modelling}

To determine the mass and radius of NGTS J0930-18 B, as well as other system parameters, we performed global fitting of both the photometric data (NGTS, TESS and SAAO) and the HARPS radial velocities. This was performed using the eclipsing binary light curve simulation code ELLC (Maxted 2016) in combination with the Markov Chain Monte Carlo (MCMC) sampler EMCEE (Foreman-Mackey et al. 2013). Before performing this, we normalised the raw lightcurves by their median out of eclipse flux, and binned the NGTS data to five minutes to reduce computational time.

The walkers were initialised in a region of parameter space which provided a good initial fit. Each walker was then given a starting position selected from a normal distribution centred on these values. We used the values derived by ORION to obtain initial values for both the transit epoch and the orbital period. Initial values for the primary star radius, stellar radius ratio, impact parameter, light ratio and radial velocity components were determined by first running the MCMC for a small number of steps to find values that gave a reasonable initial fit. We also incorporated a radial velocity jitter term added in quadrature in our modelling to account for stellar noise, as well as normalisation scaling parameters and systematic errors for each of the four lightcurves. We additionally fit for a third light parameter to account for dilution in the NGTS and TESS lightcurves. We fixed this parameter to zero for the SAAO lightcurves due to the fact that the neighbouring star was not present in the aperture used for reduction.

Limb darkening parameters were obtained using the LDTK software (Parviainen \& Aigrain 2015). A quadratic limb darkening law was used with stellar properties, e.g., $\mathrm{T}_{\text {eff }}$ and $\log g$ taken from the results given by ARIADNE. Limb darkening coefficients and uncertainties were calculated directly with LDTK, for each photometric filter used, and placed as priors for the fitting process.

An initial fit to the data resulted in an orbit with an eccentricity of $0.00592_{-0.0035}^{+0.0098}$. Lucy \& Sweeney (1971) showed that many systems with low levels of eccentricity are actually circular orbits, where the addition of eccentricity has improved the fit due to the introduction of additional free parameters. Using the methods in Lucy \& Sweeney (1971), we determine that there is an $83 \%$ probability that the measured eccentricity would be detected in a system with a true eccentricity of zero. Therefore for the final system parameters we force a circular fit.

We used 200 walkers and 50000 steps to model the lightcurve using the EMCEE sampler. Each walker used initial parameters that were randomized in a Gaussian ball around values previously determined to give a good initial fit. We note that alterations to our defined priors did not preclude the ability of the model to obtain a good fit. 10000 steps were discarded as burn-in and not used when analysing the results of the modelling. The modal values of the posterior distributions were adopted as the most probable parameters, with the 68.3 percent $(1 \sigma)$ highest probability density interval as the error estimates.

This global modeling resulted in mass and radius for NGTS J0930-18 B of $0.0818_{-0.0015}^{+0.0040} M_{\odot}\left(85.7_{-1.5}^{+4.2} M_{J}\right)$ and $0.1059_{-0.0021}^{+0.0023} R_{\odot}\left(1.052_{-0.021}^{+0.023} R_{J}\right)$. The binary has a notably low mass ratio of just $0.1407_{-0.017}^{+0.0065}$. The full list of best fitting parameters derived from the posterior distributions are given in Table 5.

\section{DISCUSSION}

\subsection{Unequal mass M-dwarf binaries}

The results from SED fitting (§3.1.3) indicate that NGTS J0930-18 A has a mass of $0.5803_{-0.0063}^{+0.0092} M_{\odot}$ and a radius of 
Table 5. Fitted and Derived parameters for NGTS J0930-18. For parameters derived using values from ARIADNE, we also report a systematic uncertainty as described in Section 3.1 .3

\begin{tabular}{|c|c|c|c|c|}
\hline Quantity & Description & unit & Value & Error \\
\hline \multicolumn{5}{|c|}{ Fitted parameters } \\
\hline$\frac{R_{\mathrm{pri}}}{a}$ & radius ratio of primary to semi-major axis & none & 0.1243 & $\begin{array}{l}+0.0023 \\
-0.0016\end{array}$ \\
\hline$k$ & radius ratio of stars, $R_{\mathrm{sec}} / R_{\mathrm{pri}}$ & none & 0.1814 & $\begin{array}{l}+0.0019 \\
-0.0020\end{array}$ \\
\hline$b$ & impact parameter, $a \cos (i) / R_{\mathrm{pri}}$ & none & 0.013 & $\begin{array}{l}+0.095 \\
-0.011\end{array}$ \\
\hline$P$ & orbital period & days & 1.33264614 & $\begin{array}{l}+0.00000126 \\
-0.00000090\end{array}$ \\
\hline$T_{c}$ & epoch of primary eclipse centre & BJD & 2457679.29957 & $\begin{array}{l}+0.00053 \\
-0.00057\end{array}$ \\
\hline$\sigma_{\mathrm{NGTS}}$ & systematic error in NGTS light curve & norm. flux & 0.00593 & $\begin{array}{l}{ }_{-0.000010}^{+0.00010} \\
-0.001\end{array}$ \\
\hline$\sigma_{I}$ & systematic error in I light curve & norm. flux & 0.00454 & $\begin{array}{l}+0.00027 \\
-0.00040\end{array}$ \\
\hline$\sigma_{g^{\prime}}$ & systematic error in g' light curve & norm. flux & 0.00838 & $\begin{array}{l}+0.00062 \\
-0.00063\end{array}$ \\
\hline$\sigma_{\mathrm{TESS}}$ & systematic error in TESS light curve & norm. flux & 0.000045 & $\begin{array}{l}+0.000330 \\
-0.000051\end{array}$ \\
\hline$\beta_{\mathrm{NGTS}}$ & normalised flux scale factor in NGTS data & none & 1.000908 & $\begin{array}{l}+0.000099 \\
-0.000016\end{array}$ \\
\hline$\beta_{I}$ & normalised flux scale factor in I data & none & 1.00086 & $\begin{array}{l}+0.00036 \\
-0.00044\end{array}$ \\
\hline$\beta_{g^{\prime}}$ & normalised flux scale factor in g' data & none & 1.00049 & $\begin{array}{l}+0.00049 \\
-0.00061\end{array}$ \\
\hline$\beta_{\text {TESS }}$ & normalised flux scale factor in TESS data & none & 1.00155 & $\begin{array}{l}+0.00030 \\
-0.00019\end{array}$ \\
\hline$u_{\text {NGTS }}$ & linear LDC in NGTS band & none & 0.499 & $\begin{array}{l}+0.035 \\
-0.026\end{array}$ \\
\hline$u_{\mathrm{NGTS}}^{\prime}$ & quadratic LDC in NGTS band & none & 0.223 & $\begin{array}{l}+0.039 \\
-0.064\end{array}$ \\
\hline$u_{\mathrm{I}}$ & linear LDC in I band & none & 0.383 & $\begin{array}{l}+0.038 \\
-0.032\end{array}$ \\
\hline$u_{\mathrm{I}}^{\prime}$ & quadratic LDC in I band & none & 0.434 & $\begin{array}{l}+0.056 \\
-0.071\end{array}$ \\
\hline$u_{\mathrm{g}^{\prime}}$ & linear LDC in g' band & none & 0.500 & ${ }_{-0.036}^{+0.029}$ \\
\hline$u_{\mathrm{g}^{\prime}}^{\prime}$ & quadratic LDC in g' band & none & 0.367 & ${ }_{-0.043}^{+0.066}$ \\
\hline$u_{\text {TESS }}$ & linear LDC in TESS band & none & 0.487 & $\begin{array}{l}+0.030 \\
-0.030\end{array}$ \\
\hline$u_{\mathrm{TESS}}^{\prime}$ & quadratic LDC in TESS band & none & 0.175 & ${ }_{-0.025}^{+0.032}$ \\
\hline$K$ & radial velocity semi-amplitude & $\mathrm{km} / \mathrm{s}$ & 21.975 & $\begin{array}{l}+0.404 \\
-0.099\end{array}$ \\
\hline$\Gamma_{\text {pri }}$ & systemic velocity & $\mathrm{km} / \mathrm{s}$ & 37.2464 & $\begin{array}{l}+0.0070 \\
-0.2443\end{array}$ \\
\hline$\sigma_{\mathrm{RV}}$ & jitter in RV data & $\mathrm{km} / \mathrm{s}$ & 0.287 & $\begin{array}{l}+0.075 \\
-0.260\end{array}$ \\
\hline \multicolumn{5}{|c|}{ Derived parameters } \\
\hline$R_{\mathrm{sec}}$ & radius of secondary & $R_{\odot}$ & 0.1059 & ${ }_{-0.0021}^{+0.0023}( \pm 0.0040)$ \\
\hline$R_{\mathrm{sec}}$ & radius of secondary & $R_{J}$ & 1.052 & ${ }_{-0.021}^{+0.023}( \pm 0.040)$ \\
\hline$m_{\mathrm{sec}}$ & mass of secondary & $M_{\odot}$ & 0.0818 & ${ }_{-0.0015}^{+0.0040}( \pm 0.0052)$ \\
\hline$m_{\mathrm{sec}}$ & mass of secondary & $M_{J}$ & 85.7 & ${ }_{-1.5}^{+4.2}( \pm 5.4)$ \\
\hline$q$ & stellar mass ratio & none & 0.1407 & ${ }_{-0.0017}^{+0.0065}( \pm 0.0085)$ \\
\hline$a$ & semi-major axis of system & $A U$ & 0.02195 & ${ }_{-0.00064}^{+0.00040}( \pm 0.00088)$ \\
\hline$i$ & orbital inclination & deg & 89.914 & ${ }_{-0.671}^{+0.085}$ \\
\hline$T_{14-p r i}$ & primary eclipse duration & hours & 1.510 & $\begin{array}{l}+0.021 \\
-0.026\end{array}$ \\
\hline
\end{tabular}

$0.584_{-0.010}^{+0.0094} R_{\odot}$ i.e. an early M-dwarf (Kaltenegger \& Traub 2009). Thus the system NGTS J0930-18 is an unequal mass eclipsing M-dwarf binary. This is an unusual configuration, as most known eclipsing M-dwarf systems (e.g Parsons et al. 2018) are in near equal mass binaries. With a mass ratio of $0.1407_{-0.017}^{+0.0065}$ NGTS J0930-18 is highly unusual. The mass ratio is more similar to the well-studied population of low mass M-dwarfs in orbit around F- G- K- stars (e.g., Triaud et al. 2017) or transiting brown dwarf systems around early/mid M-dwarfs (e.g., Irwin et al. 2010; Johnson et al. 2011; Montet et al. 2015; Gillen et al. 2017; David et al. 2019).

It is expected that short-period M-dwarf systems with unequal mass components will be very rare. During formation, mass is preferentially accreted onto the lower mass component of the binary (Bate et al. 2002) which will over time drive the mass ratio towards unity and result in a system with roughly equal mass components. For short period, low mass systems this effect is expected to be even greater due to dynamical effects. An early post-collapse star forming cloud will fragment into a low number of multiple systems (Goodwin \& Whitworth 2007). Dynamical decay and interactions within this collapsing cloud are biased against low mass components, which are typically ejected on a short timescale (Anosova 1986). In interactions with higher mass stars this means the low mass star tends to be swapped with 

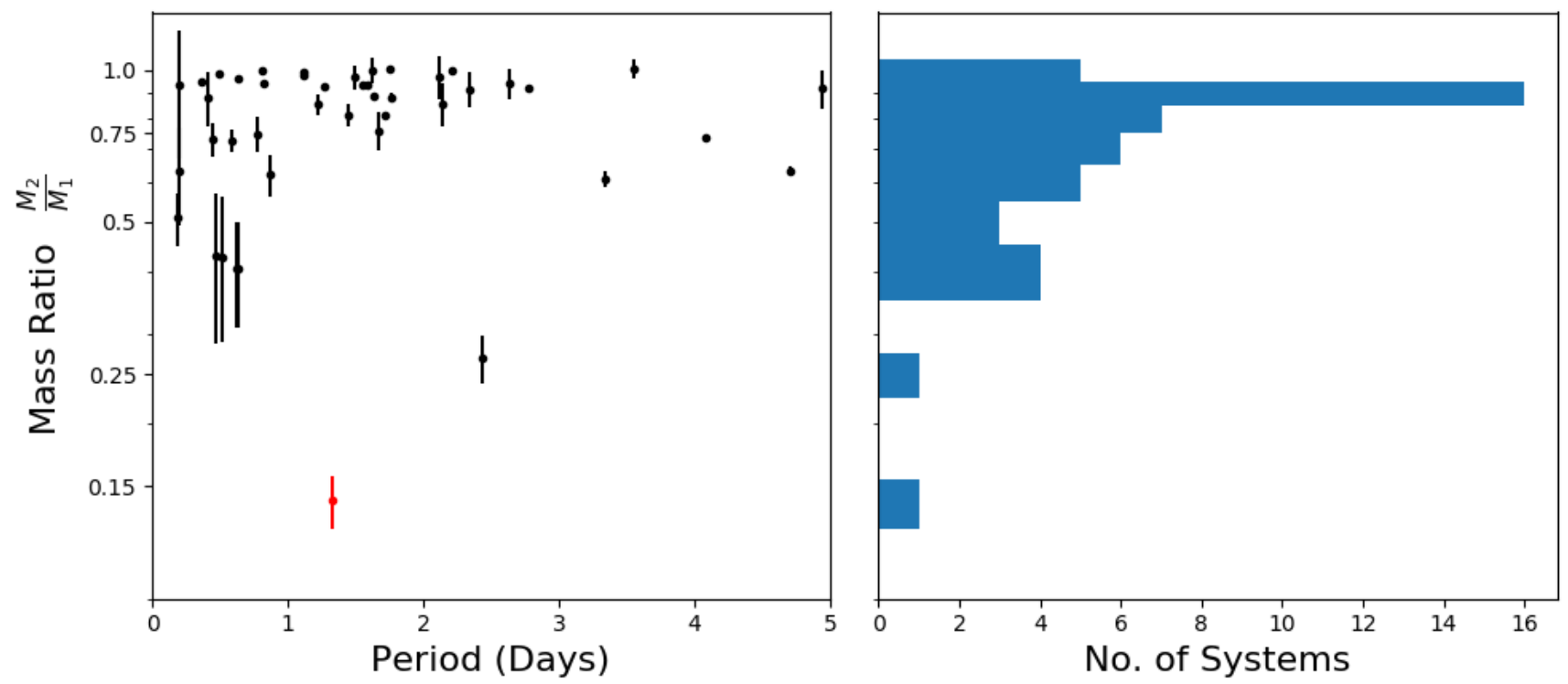

Figure 9. Left - Mass ratio as a function of period for M-dwarf binaries given by Parsons et al. 2018 and Nefs et al. 2013. NGTS J0930-18 is indicated in Red. Right - Histogram showing the distribution of M-dwarf binary mass ratios. NGTS J0930-18 is a clear outlier from the general population.

a higher mass replacement. Thus the mechanisms that produce short period binaries are biased to produce equal mass systems.

This theory is supported by observational evidence. Delfosse et al. 2004 surveyed a large number of M-dwarf binaries in the Solar neighborhood. They found that for systems with a period less than 50 days, the distribution of the mass ratio peaked close to unity. The orbital period of NGTS J0930-18 is significantly shorter than this at 1.33265 days, meaning its formation and survival probes a sparse area of binary star parameter space. There are, however, examples of similar unequal mass binaries in the literature (e.g., Nefs et al. 2013).

In Figure 9 we show the mass ratio as a function of period for short period (less than 5 days) systems with Mdwarf primaries taken from the samples in Parsons et al. 2018 and Nefs et al. 2013. From Figure 9 it is clear that NGTS J0930-18 has the lowest mass ratio of any known Mdwarf binary system. This also demonstrates that the vast majority of short period systems of this type have a mass ratio close to 1 , given the large cluster of systems in the top left of the plot. Such a short period system of two M-dwarfs with highly unequal masses is clearly unusual.

\subsection{M-dwarf Mass-Radius Relationship}

The global modelling performed in section 3.2 indicates NGTS J0930-18 B has a mass of $85.7_{-1.5}^{+4.2} \mathrm{M}_{\mathrm{J}}$. This places it just above the hydrogen burning mass limit of $\sim 70 \mathrm{MJ}_{\mathrm{J}}$ which separates brown dwarfs and low mass stars (Baraffe et al. 1998). The mass and radius of the star are similar to that of TRAPPIST-1 (Gillon et al. 2017), demonstrating the importance of precise measurements in this parameter space to characterise current and future exoplanet discoveries.

In Figure 10 we compare the mass and radius of NGTS

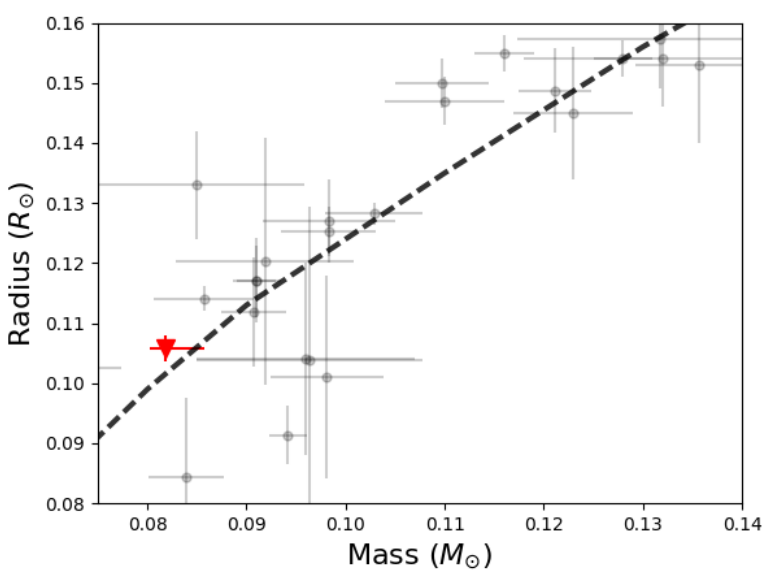

Figure 10. Comparison between NGTS J0930-18 B and a model stellar isochrone from Baraffe et al. 2015. The 10-Gyr isochrone is indicated by the black dashed line, and NGTS J0930-18 B by the red triangle. Similar M-dwarfs from Parsons et al. 2018, Triaud et al. 2020 and Mireles et al. 2020 are shown in Black.

J0930-18 B to a sample of low mass stars from Parsons et al. 2018, as well as a model 10-Gyr stellar isochrone from Baraffe et al. 2015. Figure 10 illustrates the low number of systems known in this parameter space. Most of the systems are from detections of low mass secondary stars in eclipsing binaries with higher mass primaries, the same configuration as NGTS J0930-18. We also note the well-known scatter in the mass-radius relationship for low mass stars is still present even at the lower end of the mass distribution.

This shows that the radius of NGTS J0930-18 B is very slightly inflated relative to models, but is still consistent to 
1-sigma for its mass. Inflated radii of low mass stars can often be associated with short orbital periods (Spada et al. 2013). This is due to the fact that at fast rotation speeds magnetic activity is enhanced which can affect convective processes, causing an inflation in stellar radius (López-Morales 2007; Chabrier et al. 2007). However inflation of stellar radii has also been seen in longer period systems (e.g., Acton et al. 2020) indicating that this deviation is not consistent. Indeed, with a period of just 1.33265 days we may have expected this object to show some level of inflation, which is not clearly seen. We note that the HARPS spectrum of the star shows clear $\mathrm{H} \alpha$ emission, a strong indicator that the star is indeed magnetically active.

We note that direct detection of the secondary eclipse of this system to refine these parameters in the future will likely be difficult. The non detection of a secondary eclipse in the NGTS data can be used to place an upper limit on the light ratio of the two stars. However given the out of eclipse scatter a secondary eclipse would still not be detected even if NGTS J0930-18 B was more luminous than it actually is. For a $0.08 M_{\odot}$ dwarf with an age of $10 \mathrm{Gyr}$, Baraffe et al. (2015) give a temperature of 2345 K. From determining the surface brightness ratio for the system given the temperature of the primary determined in Section 3.1.3, we find the secondary eclipse depth to be around $0.25 \%$, well within the scatter of both the TESS and NGTS lightcurves. An eclipse this shallow will be difficult to detect given the faintness of the system. Spectroscopic detection of a second set of lines associated with the secondary will also be difficult due to the rapid rotation of the star resulting in the blending of the spectral lines.

It is also important to note the precise mass and radius measurements obtained for NGTS J0930-18 B when compared with similar objects in Figure 10. Owing to the high precision photometry and radial velocities used to characterise the system we have derived the mass and radius of NGTS J0930-18 B to a precision of $5 \%$ and $2 \%$ respectively. High precision measurements in this regime are vital for the empirical derivation of the mass-radius relationship stars at the lowest end of the mass radius distribution.

\section{CONCLUSIONS}

We have discovered the M-dwarf eclipsing binary system NGTS J0930-18, with the secondary component, NGTS J0930-18 B, having a mass just above the hydrogen burning limit. We were able to determine a very precise mass and radius for NGTS J0930-18 B, and these parameters are of great scientific interest due to the prominence of very low mass stars in the search for temperate terrestrial exoplanets. Knowledge of the masses and radii of these stars are vital for characterisation of future exoplanet discoveries, and these measurements can only be obtained precisely through the characterisation of eclipsing binary stars similar to this system. NGTS J0930-18 B provides a valuable data point in a sparsely populated region of parameter space and will be of importance for future work in empirically deriving the mass-radius relationship for the lowest mass stars.

\section{ACKNOWLEDGEMENTS}

Based on data collected under the NGTS project at the ESO La Silla Paranal Observatory. The NGTS facility is operated by the consortium institutes with support from the UK Science and Technology Facilities Council (STFC) under projects ST/M001962/1 and ST/S002642/1.

This paper includes data collected by the TESS mission. Funding for the TESS mission is provided by the NASA Explorer Program.

This paper uses observations made at the South African Astronomical Observatory (SAAO). We thank Marissa Kotze (SAAO) for developing the SHOC camera data reduction pipeline.

This study is based on observations collected at the European Southern Observatory under ESO programme 0103.C-0719.

JA is supported by an STFC studentship. BTG, SG and PJW acknowledge support from STFC consolidated grants ST/L000733/1 and ST/P000495/1.MNG acknowledges support from MIT's Kavli Institute as a Juan Carlos Torres Fellow. JSJ acknowledges support by FONDECYT grant 1201371, and partial support from CONICYT project Basal AFB-170002.EG gratefully acknowledges support from the David and Claudia Harding Foundation in the form of a Winton Exoplanet Fellowship.

We thank the anonymous referee for their useful and constructive feedback to improve the paper.

\section{DATA AVAILABILITY}

The data underlying this article will be shared on reasonable request to the corresponding author.

\section{REFERENCES}

Acton J. S., et al., 2020, MNRAS, 494, 3950

Allard F., Homeier D., Freytag B., 2012, Philosophical Transactions of the Royal Society A: Mathematical, Physical and Engineering Sciences, 370, 2765

Anosova J. P., 1986, Ap\&SS, 124, 217

Baraffe I., Chabrier G., Allard F., Hauschildt P. H., 1998, A\&A, 337,403

Baraffe I., Homeier D., Allard F., Chabrier G., 2015, A\&A, 577, A42

Bate M. R., Bonnell I. A., Bromm V., 2002, MNRAS, 332, L65

Bayliss D., et al., 2018, MNRAS, 475, 4467

Blake C. H., Torres G., Bloom J. S., Gaudi B. S., 2008, ApJ, 684, 635

Blanco-Cuaresma S., Soubiran C., Heiter U., Jofré P., 2014, A\&A, 569, A111

Bouchy F., et al., 2011, A\&A, 533, A83

Casewell S. L., et al., 2018, MNRAS, 481, 1897

Castelli F., Kurucz R. L., 2004, preprint, (arXiv:0405087)

Chabrier G., Gallardo J., Baraffe I., 2007, A\&A, 472, L17

Choi J., Dotter A., Conroy C., Cantiello M., Paxton B., Johnson B. D., 2016, ApJ, 823, 102

Coppejans R., et al., 2013, PASP, 125, 976

Covey K. R., et al., 2007, AJ, 134, 2398

David T. J., Hillenbrand L. A., Gillen E., Cody A. M., Howell S. B., Isaacson H. T., Livingston J. H., 2019, ApJ, 872, 161

Delfosse X., et al., 2004, M dwarfs binaries: Results from accurate 
radial velocities and high angular resolution observations. pp 166-174

Delrez L., et al., 2018, in Proc. SPIE. p. $107001 \mathrm{I}$ (arXiv: 1806.11205), doi:10.1117/12.2312475

Dieterich S. B., Henry T. J., Jao W.-C., Winters J. G., Hosey A. D., Riedel A. R., Subasavage J. P., 2014, AJ, 147, 94

Doyle L. R., et al., 2011, Science, 333, 1602

Feiden G. A., Chaboyer B., 2012, ApJ, 757, 42

Foreman-Mackey D., Hogg D. W., Lang D., Goodman J., 2013, PASP, 125, 306

Gaia Collaboration et al., 2018, A\&A, 616, A1

Gill S., et al., 2019a, MNRAS, p. 2805

Gill S., et al., 2019b, Astronomy \& Astrophysics, 626, A119

Gill S., et al., 2020, ApJ, 898, L11

Gillen E., Hillenbrand L. A., David T. J., Aigrain S., Rebull L., Stauffer J., Cody A. M., Queloz D., 2017, ApJ, 849, 11

Gillon M., Jehin E., Magain P., Chantry V., Hutsemékers D., Manfroid J., Queloz D., Udry S., 2011, in European Physical Journal Web of Conferences. p. 06002 (arXiv: 1101.5807), doi:10.1051/epjconf/20101106002

Gillon M., et al., 2017, Nature, 542, 456

Goodwin S. P., Whitworth A., 2007, A\&A, 466, 943

Günther M. N., et al., 2017, MNRAS, 472, 295

Günther M. N., et al., 2019, Nature Astronomy, p. 420

GÃşmez Maqueo Chew Y., et al., 2014, Astronomy \& Astrophysics, 572, A50

Hauschildt P. H., Allard F., Baron E., 1999, ApJ, 512, 377

Henry T. J., Jao W.-C., Subasavage J. P., Beaulieu T. D., Ianna P. A., Costa E., Méndez R. A., 2006, AJ, 132, 2360

Husser T.-O., Wende-von Berg S., Dreizler S., Homeier D., Reiners A., Barman T., Hauschildt P. H., 2013, A\&A, 553, A6

Irwin J., et al., 2010, ApJ, 718, 1353

Irwin J. M., et al., 2011, ApJ, 742, 123

Jackman J. A. G., et al., 2019, MNRAS, 489, 5146

Johnson J. A., et al., 2011, ApJ, 730, 79

Kaltenegger L., Traub W. A., 2009, The Astrophysical Journal, 698, 519âĂŞ527

Kesseli A. Y., West A. A., Veyette M., Harrison B., Feldman D., Bochanski J. J., 2017, ApJS, 230, 16

Kostov V. B., et al., 2019, arXiv e-prints,

Kovács G., Zucker S., Mazeh T., 2016, BLS: Box-fitting Least Squares, Astrophysics Source Code Library (ascl:1607.008)

Kraus A. L., Tucker R. A., Thompson M. I., Craine E. R., Hillenbrand L. A., 2011, ApJ, 728, 48

Kurucz R. L., 1993, VizieR Online Data Catalog, 6039

Laithwaite R. C., Warren S. J., 2020, arXiv e-prints, p. arXiv:2006.11092

Lendl M., et al., 2019, MNRAS, p. 3189

López-Morales M., 2007, ApJ, 660, 732

Lucy L. B., Sweeney M. A., 1971, AJ, 76, 544

Maxted P. F. L., 2016, A\&A, 591, A111

Mayor M., et al., 2003, The Messenger, 114, 20

Mireles I., et al., 2020, arXiv e-prints, p. arXiv:2006.14019

Montet B. T., et al., 2015, ApJ, 800, 134

Nefs S. V., et al., 2013, Monthly Notices of the Royal Astronomical Society, 431, 3240

Parsons S. G., et al., 2018, MNRAS, 481, 1083

Parviainen H., Aigrain S., 2015, MNRAS, 453, 3821

Ribas I., 2006, Ap\&SS, 304, 89

Ricker G. R., et al., 2014, in Proc. SPIE. p. 914320 (arXiv: 1406.0151), doi:10.1117/12.2063489

Schlafly E. F., Finkbeiner D. P., 2011, Astrophysical Journal, 737 Schlegel D. J., Finkbeiner D. P., Davis M., 1998, ApJ, 500, 525

Skrutskie M. F., et al., 2006, AJ, 131, 1163

Southworth J., et al., 2015, MNRAS, 454, 3094

Spada F., Demarque P., Kim Y. C., Sills A., 2013, ApJ, 776, 87

Speagle J. S., 2019, arXiv e-prints, p. arXiv:1904.02180

Stassun K. G., et al., 2019, AJ, 158, 138
Stevens D. J., et al., 2019, arXiv e-prints, p. arXiv:1910.06212

Tamuz O., Mazeh T., Zucker S., 2005, MNRAS, 356, 1466

Terrien R. C., Fleming S. W., Mahadevan S., Deshpande R., Feiden G. A., Bender C. F., Ramsey L. W., 2012, ApJ, 760, L9

Triaud A. H. M. J., et al., 2012, Astronomy \& Astrophysics, 549, A18

Triaud A. H. M. J., et al., 2017, Astronomy \& Astrophysics, 608, A129

Triaud A. H. M. J., et al., 2020, Nature Astronomy, 4, 650

Vines J. I., Jenkins J. S., 2020, in prep

Wheatley P. J., et al., 2018, MNRAS, 475, 4476

Wisniewski J. P., et al., 2012, AJ, 143, 107

Yee S. W., Petigura E. A., von Braun K., 2017, ApJ, 836, 77

von Boetticher A., et al., 2017, Astronomy \& Astrophysics, 604, L6

von Boetticher A., et al., 2019, Astronomy \& Astrophysics, 625, A150

This paper has been typeset from a $\mathrm{T}_{\mathrm{EX}} / \mathrm{LAT}_{\mathrm{EX}}$ file prepared by the author. 